\title{
Optimisation and molecular signalling of apoptosis in sequential cryotherapy and chemotherapy combination in human A549 lung cancer xenografts in SCID mice
}

\author{
V Forest ${ }^{*, 1}$, R Hadjeres ${ }^{2}$, R Bertrand ${ }^{1,4}$ and R Jean-François ${ }^{3,4}$ \\ 'Centre de recherche and Institut du cancer de Montréal, Montréal, Canada; ²Département de pathologie, Centre Hospitalier de l'Université de Montréal \\ (CHUM)-Hôpital Notre-Dame, Montréal (QC) H2L 4MI, Canada; ${ }^{3}$ Département de pneumologie, Centre Hospitalier de l'Université de Montréal \\ (CHUM)-Hôpital Notre-Dame, Montréal (QC) H2L 4MI, Canada; ${ }^{4}$ Département de Médecine, Université de Montréal, Montréal (QC) H3C 3J7, \\ Canada
}

We define the optimal parameters for combination of cryotherapy (nitrous oxide) with chemotherapy (vinorelbine ditartrate, VNB) treatment and characterise some of the signals involved for apoptosis activation. No advantage appeared when cryotherapy and VNB were combined simultaneously compared to cryosurgery alone. In contrast, tumour volumes were reduced after a sequential treatment schedule, where each individual treatment was separated by $48 \mathrm{~h}$. No significant benefit appeared when the sequential treatment was separated by $24 \mathrm{~h}$, although some individual mice showed a good response. The sequence of treatment had no impact on the observed tumour growth inhibition in mice. The number of apoptotic cells was significantly augmented in the sequential treatment schedule where VNB was administered $48 \mathrm{~h}$ before cryotherapy. In this sequential treatment, the number of apoptotic cells correlated with heightened expression of the BH3-only Puma, Noxa and Bim-EL, at both the mRNA and protein levels. No significant change in $\mathrm{Bax}, \mathrm{Bcl}-\mathrm{xL}$ and $\mathrm{Bcl}-2$ mRNA expression was apparent, whereas $\mathrm{Mcl}-\mathrm{I}$ expression increased only slightly to a much lower level than $\mathrm{BH}$-only mRNAs. Our data indicate that $48 \mathrm{~h}$ sequential rather than simultaneous cryotherapy with VNB in future cancer cryochemotherapy schedules will enhance the tumour response, and argue that VNB administration, $48 \mathrm{~h}$ before cryotherapy, will provoke apoptosis more efficiently.

British Journal of Cancer (2009) I 00, 1896-1902. doi:I0.1038/sj.bjc.6605046 www.bjcancer.com

Published online 19 May 2009

(c) 2009 Cancer Research UK

Keywords: cryotherapy; chemotherapy; combination treatment; lung cancer; Bcl-2 family

Cryosurgery, a treatment based on the cytotoxic effects of cold, consists of therapeutically applying extremely low temperatures to living tissues to destroy them. It represents a minimally invasive surgical technique that has expanded in applicability in recent years. In the treatment of lung cancers, it can be proposed as a palliative option, mainly to relieve airway obstruction and improve the patient's respiratory functions. It is safe, efficient, inexpensive, easy to perform and does not present any major side effect (Maiwand and Homasson, 1995; Vergnon, 1999). Although cryosurgery is a potent method of in situ tissue destruction, it generally needs the support of adjunctive therapy, including chemotherapy or radiotherapy, to increase the rate of cell death in the peripheral zone of the cryolesion. In this area, less damaged cells can repair themselves and survive, requiring further injury to synergise lethality (Gage and Baust, 1998; Korpan, 2001; Baust et al, 2004). Moreover, it is well known that vascular changes occur in tissues after freezing, and it is assumed that the hypervascularity observed enhances tissue radiosensitivity and allows a better drug delivery to tumour cells (Vergnon, 1999).

*Correspondence: Dr V Forest; E-mail: valerie.forest. I@umontreal.ca Received 23 January 2009; revised 25 March 2009; accepted 25 March 2009; published online 19 May 2009
In vitro, combination of cryotherapy and chemotherapy, also so-called cryochemotherapy, has delivered promising results. Mir and Rubinsky (2002) have reported increased cytotoxicity in melanoma cells subjected to cryochemotherapy. The cells were first exposed to $-20^{\circ} \mathrm{C}$ before bleomycin treatment and compared to cells treated only with bleomycin. The effect was due to cold-induced membrane disorganisation, allowing greater bleomycin influx into cells. A similar advantage of cryochemotherapy was noted in $\mathrm{PC}-3$ prostate cancer cells (Clarke et al, 2001). In vivo, Le Pivert et al (2004) showed beneficial actions of cryosurgery and local chemotherapy in a model of human prostate tumour xenografts in nude mice. In this study, a single freeze/thaw cycle was applied to tumours, and 5-fluorouracil (5-FU) microcapsules were delivered at the outer margins of frozen areas. This combined treatment produced better inhibition of tumour growth for a longer period of time than cryoablation or local 5-FU microcapsule administration alone. Earlier, we also reported a model of human A549 lung adenocarcinoma xenografts in SCID mice, where combination of cryotherapy (nitrous oxide) with chemotherapy (vinorelbine ditartrate; VNB) enhanced cell death by necrosis and apoptosis, mainly during the early phase of treatments, and limited tumour growth, as tumours subjected to cryochemotherapy presented a significantly reduced volume compared with tumours 
undergoing cryosurgery or chemotherapy alone (Forest et al, 2005a,b; Forest et al, 2006).

A wide range of current studies focus particularly on how cell death occurs after cryosurgery. It has been well documented that apoptosis is an important mechanism of cell death when temperature does not fall low enough to kill cells through direct ice rupture or necrosis (Gage and Baust, 1998; Baust et al, 2004). However, the molecular mechanisms involved are not elucidated. The Bcl-2 family of proteins stands among the more important regulators of apoptosis, and consists of both pro- and antiapoptotic members, which are characterised by the presence of different $\mathrm{BH}$ (Bcl-2 homology) domains (Adams and Cory, 2007). The ratio between the proapoptotic and the prosurvival members helps to determine, in part, the susceptibility of cells to apoptosis (Korsmeyer et al, 1993). In addition, a subset of this family, the BH3-only proteins, has been well recognised as cell death signal transmitters and potent mediators of apoptosis. Among them, Puma, Noxa and Bim are often associated with chemotherapyinduced apoptosis (Oda et al, 2000; Yu et al, 2001; Willis et al, 2007).

The aims of this study were (1) to define the optimal parameters of combined cryotherapy and VNB treatment in a model of lung adenocarcinoma xenografted into SCID mice and (2) to characterise the molecular signalling of apoptosis after these treatment schedules. For these purposes, we compared the ability of different treatment protocols to induce cell death and investigated the involvement of some Bcl-2 family members, including Puma, Noxa, Bim-EL, Bax, Bcl-2, Bcl-xL and Mcl-1.

\section{MATERIALS AND METHODS}

\section{Experimental model}

The A549 cell line, derived from a human lung adenocarcinoma, was purchased from the American Type Culture Collection (Manassas, VA, USA). The cells were maintained in culture at $37^{\circ} \mathrm{C}$ in a humidified $5 \% \mathrm{CO}_{2}$ atmosphere, in Ham's F-12 medium containing 2 mM L-glutamine (Gibco-BRL Life Technologies, Grand Island, NY, USA) and supplemented with $10 \%$ foetal bovine serum (Wisent Inc., Montreal, QC, Canada), $1.5 \mathrm{gl}^{-1}$ sodium bicarbonate (Gibco-BRL Life Technologies), $10000 \mathrm{U} \mathrm{ml}^{-1}$ penicillin (Wisent Inc.) and $10 \mathrm{mg} \mathrm{ml}^{-1}$ streptomycin (Wisent Inc.). Male SCID mice (2-3 weeks old, $22-24 \mathrm{~g}$ body weight) were supplied by Charles River Laboratory Inc. (Wilmington, MA, USA) and housed in sterile cages. They were fed autoclaved food and sterile water ad libitum. For inoculation into SCID mice, the A549 cells were trypsinised, washed and resuspended in sterile phosphate-buffered saline (PBS, Wisent Inc.). The mice were injected subcutaneously with this suspension $\left(10^{7}\right.$ cells in $\left.0.2 \mathrm{ml}\right)$, in the dorsal region, bilaterally. When the tumours reached a volume of about $0.8 \mathrm{~cm}^{3}$, the mice were treated. All operative procedures and animal care conformed strictly to Canadian Council on Animal Care guidelines.

\section{Cryosurgery and chemotherapy}

Eight experimental groups of three mice each were allocated depending on the control or treatment regimen: (1) no treatment (control group), (2) cryotherapy only (cryotherapy group), (3) chemotherapy only (chemotherapy group), (4) cryotherapy and chemotherapy simultaneously (cryochemotherapy group), (5) cryotherapy followed by chemotherapy after $24 \mathrm{~h}$ (cryo/24/ chemotherapy group), (6) chemotherapy followed by cryotherapy after $24 \mathrm{~h}$ (chemo/24/cryotherapy group), (7) cryotherapy followed by chemotherapy after $48 \mathrm{~h}$ (cryo/48/chemotherapy group) and (8) chemotherapy followed by cryotherapy after $48 \mathrm{~h}$ (chemo/48/ cryotherapy group). Cryotherapy was carried out with a nitrous oxide cryoprobe, $3 \mathrm{~mm}$ in diameter (Erbe Inc., Tübingen, Germany). The Joule-Thomson effect allowed us to attain temperatures ranging from -30 to $-40^{\circ} \mathrm{C}$ in tissues. After an incision was made in the tumour, the cryoprobe was placed in contact with it for three cycles of rapid freezing/thawing (20/20 s each). Chemotherapy consisted of a single intravenous injection of VNB (Navelbine, Mayne Pharma Inc., Montreal, QC, Canada), a vinca alkaloid commonly used in lung cancer treatment. A clinically equivalent dose $\left(4.8 \mathrm{mg} \mathrm{kg}^{-1}\right)$ was injected into the tail vein of mice previously anesthetised with isoflurane $2 \%$. The animals were then euthanised at variable time points before (control) and after therapy, at 2, 8, 24, 48 h, 4, 8, 14 and 21 days ( $n=3$ for each point), and then tumours were excised. They were sliced along the axis of the cryoprobe impact site. One-half of the tumours was frozen directly in liquid nitrogen and kept for future RNA extraction, and the other half was frozen in liquid nitrogen but in the presence of optimal cutting temperature medium (Ted Pella Inc., Redding, CA, USA) for future morphological and immunohistochemical staining.

\section{Kinetics of tumour growth}

To evaluate the effects of the different treatments on tumour growth, tumour volume (TV) was determined as follows: TV $=L \times$ $W \times H$, where $L$ was the length, $W$ the width and $H$ the height of the tumour, measured with a caliper. Relative tumour volume (RTV) was then quantitated according to the formula RTV $=\mathrm{TV}_{\mathrm{x}} I$ $\mathrm{TV}_{0}$, where $\mathrm{TV}_{\mathrm{x}}$ corresponds to TV at day $\mathrm{x}$, and $\mathrm{TV}_{0}$ to $\mathrm{TV}$ at day 0 , before treatment.

\section{Immunohistochemical staining}

Frozen sections were exposed to standard haematoxylin-eosinsaffron coloration to analyse tissue morphology. Necrosis, estimated by a clinical pathologist in terms of a ratio between the surface of necrotic areas and that of the whole tumour, was expressed as percentages. Other tissue sections were fixed and permeabilised by incubation in a $50 \%$ acetone - 50\% methanol solution for $3 \mathrm{~min}$ at room temperature. Tissue sections were washed with PBS and stained in Vectastain ABC-Alkaline Phosphatase Universal and Alkaline Phosphatase Substrate kits (Vector Laboratories Inc., Burlingame, CA, USA), according to the manufacturer's instructions. The primary antibodies used were anti-active caspase- 3 rabbit pAb 559565 (BD Biosciences Pharmingen, Mississauga, ON, Canada) at $1: 1000$ dilution, antiPuma rabbit pAb PC686 (EMD-Biosciences-Calbiochem Inc., La Jolla, CA, USA) at 1:50 dilution, anti-Noxa mouse mAb 114C307 (EMD-Biosciences-Calbiochem Inc.) at 1:50 dilution, anti-Bim rabbit pAb 202000 (BD Biosciences Pharmingen) at 1:50 dilution and anti-Bax mouse mAb Ab-2 (EMD-Oncogene Research Inc., San Diego, CA, USA) at 1:50 dilution. After staining, slices were washed with PBS, mounted with Vectashield (Vector Laboratories Inc.) and images were generated with a Nikon Optiphot-2 microscope equipped and mounted with a thermoelectrically cooled CCD camera (Model DC330E, DageMTI Inc., Michigan City, IN, USA) hooked up to a PC computer. Images were analysed with Clemex Vision software (Version 3.0.036, Clemex, Longueuil, QC, Canada), and quantitative data on caspase- 3 active fragment were based on integrated volume values defined as intensity $(\%) \times$ area $\left(\mu \mathrm{m}^{2}\right)$.

RNA isolation, reverse transcription and quantitative real-time polymerase chain reaction ( $Q R T$ - PCR)

RNA was extracted from frozen tissues in Trizol reagent (Invitrogen Corp., Burlington, ON, Canada) according to the manufacturer's instruction. cDNA was synthesised with $1 \mu \mathrm{g}$ RNA 
per sample by the SuperScript first-strand synthesis system (Invitrogen Corp.). The cDNA was then amplified by QRT-PCR in Rotor Gene (Model RG-3000A, Corbett Res., Mortlake, Australia) with QuantiTect SYBR Green kits (Qiagen, Missisauga, ON, Canada) in a final volume of $25 \mu \mathrm{l}$. The primer pairs were Puma: 5'-TGGA CTCAGCATCGGAAGGT- $3^{\prime}$ (forward), 5'-GCACCAGCACAACAG CCTTT-3' (reverse), Noxa: 5'-TTCGTGTTCAGCTCGCGTCC-3' (forward), $5^{\prime}$-CTCGGTTGAG CGTTCTTGCG-3' (reverse), Bim-EL: $5^{\prime}$-TGATGTAAGTTCTGAAGTGTG-3' (forward), $5^{\prime}$-CTGGGAGGA TCTTCTCATAA- $3^{\prime}$ (reverse), Bax: $5^{\prime}$-AACTGGTGCTCAAGGCC CTG-3' (forward), 5'-GGGTGAGGAGGCTTGAGGAG-3' (reverse), Bcl-2: $5^{\prime}$-TTTGAGTTCG GTGGGGTCATG-3' (forward), 5'-TCACTT GTGGCTCAGATAGGC-3' (reverse), Bcl-xL: 5'-ATCAATGGCAACC CATCCTGG- $3^{\prime}$ (forward), $5^{\prime}$-TTGTCTACGCTTTCCACGCAC- ${ }^{\prime}$ (reverse), Mcl-1: 5'-GCTGCATCGAACCATTAGCAG-3' (forward), $5^{\prime}$-TATGCCAAACC AGCTCCTACT-3' (reverse), $\beta$-actin: $5^{\prime}$-ACTCT TCCAGCCTTCCTTCC-3' (forward), $5^{\prime}$-GTACTTGCGCTCAGGAGG AG- $3^{\prime}$ (reverse). Cycling conditions were initiated with denaturation for $15 \mathrm{~min}$ at $95^{\circ} \mathrm{C}$, followed by 40 cycles of denaturation $\left(95^{\circ} \mathrm{C}\right.$ for $15 \mathrm{~s}$ ), annealing $\left(58^{\circ} \mathrm{C}\right.$ for Puma, $59^{\circ} \mathrm{C}$ for Noxa and Bax, $51^{\circ} \mathrm{C}$ for Bim-EL, $60^{\circ} \mathrm{C}$ for $\mathrm{Bcl}-2$, Bcl-xl and $\mathrm{Mcl}-1,30 \mathrm{~s}$ ) and extension $\left(72^{\circ} \mathrm{C}\right.$ for $\left.30 \mathrm{~s}\right)$. Messenger RNAs (mRNAs) were quantitated according to the Pfaffl mathematical model (Pfaffl, 2001). The amount of targeted mRNAs was normalised to endogenous reference mRNA ( $\beta$-actin), and the data on treated tumours were expressed relative to untreated tumours at day 0 . The results are the means of three independent experiments, each carried out in duplicate.

\section{Statistical analysis}

Statistical analyses were carried out by two-way analysis of variance with GraphPad Prism software (version 4.0c; San Diego, CA, USA) and $P>0.05$ values were considered as non-significant (n.s.). All statistical analyses are provided online in the Supplemental Data section.

\section{RESULTS}

\section{Kinetics of tumour growth}

This study compared the kinetics of tumour growth after individual, simultaneous and sequential combination of cryotherapy and VNB treatment, in human A549 lung adenocarcinoma xenograft-bearing SCID mice (Figure 1 and Supplementary Data).

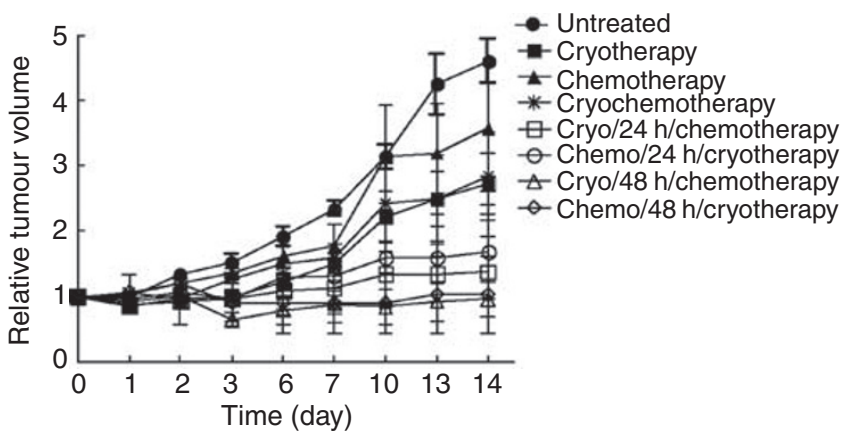

Figure I Kinetics of tumour growth after individual, simultaneous and sequential combination of cryotherapy and VNB treatment in human A549 lung adenocarcinoma xenograft-bearing SCID mice. Relative tumour volumes (y axis) were measured as described in Materials and Methods at various times ( $x$ axis) after the end of treatments. Points and vertical bars represent the means \pm s.e.m. of three independent experiments (three mice). Statistical analyses are provided in Supplementary Data.
At the concentration $\left(4.8 \mathrm{mg} \mathrm{kg}^{-1}\right)$ used in this study, the slight difference observed in RTVs of mice treated with VNB only compared with untreated controls was not statistically significant. In contrast, cryotherapy alone produced a significant effect, with RTVs much reduced than in untreated mice. Simultaneous exposures to cryotherapy and VNB (cryochemotherapy) achieved a very similar outcome on tumour growth as in mice subjected only to cryotherapy. RTVs in mice undergoing cryotherapy alone or simultaneous combination appeared stable for 3 days posttreatment, but increased steadily thereafter, indicating that the actions of these therapies were only partial. In the sequential combination schedules, a marked and significant effect on tumour growth was apparent when each treatment was separated by $48 \mathrm{~h}$. Although some individual mice showed a good response when each individual treatment was separated by $24 \mathrm{~h}$, the overall differences between these groups and the simultaneous combination schedule were not statistically significant. Moreover, the sequence of each treatment in these sequential combination protocols had no major influence on the kinetics of tumour growth inhibition. Interestingly, the $48 \mathrm{~h}$ sequential combination schedules also achieved a strong inhibition of tumour growth in mice for a longer period of time. All statistical analyses are provided in Supplementary Data.

\section{Cell death induction}

To investigate the mode and relative amount of cell death after the different treatments and schedules, morphological assignment of necrosis was blind-performed by a pathologist experienced in Haematoxylin-Eosin-Saffron staining of tumour samples with routinely deployed clinical criteria, and apoptosis was determined by the presence of active caspase-3 proteolytic fragment under immunohistochemical staining. Despite individual variations within mice, the relative amount of necrosis was generally low, ranging from 5 to $35 \%$, and changes among the various combination protocols and time of analysis were not significant (Figure 2A and Supplementary Data). Similarly, no strong difference in the amount of apoptosis was observed among most combination protocols tested, except when VNB was administered $48 \mathrm{~h}$ before cryotherapy (Figure $2 \mathrm{~B}$ and Supplementary Data). In this sequential treatment protocol, the amount of apoptotic cells was significantly enhanced after $48 \mathrm{~h}$ of combined treatment, indicating heightened apoptotic activities in these tumours.

\section{Molecular signalling of apoptosis}

To further investigate the molecular signalling of apoptosis in these tumours, attention was focused on $\mathrm{Bcl}-2$ family members. First, we undertook QRT - PCR and evaluated the relative mRNA expression level of some proapoptotic members, including the BH3-only Puma, Noxa and Bim-EL, and multidomain Bax members. Strikingly, the expression of Puma, Noxa and Bim-EL mRNAs increased significantly $24 \mathrm{~h}$ and $48 \mathrm{~h}$ post-treatment in the sequential combination schedule, where VNB was given $48 \mathrm{~h}$ before cryotherapy (Figure $3 \mathrm{~A}$ and Supplementary Data). The kinetics of expression of these genes were correlated with the augmented amount of apoptotic cells seen in tumours treated with this sequential combination schedule (Figure 2B). Messenger RNA levels of Bax and of the antiapoptotic Bcl-xL (Figure 3B) and Bcl-2 (data not shown) did not change dramatically among all tumours tested, whereas Mcl-1 mRNA expression showed a small yet significant increase, but at a much lower level than the BH3-only Puma, Noxa and Bim-EL (Figure 3B and Supplementary Data). These observations were confirmed by immunohistochemical staining analysis, where elevated expression of Puma, Noxa and Bim, but not Bax proteins could be easily visualised in tumour samples obtained $48 \mathrm{~h}$ post-treatment from mice treated with 

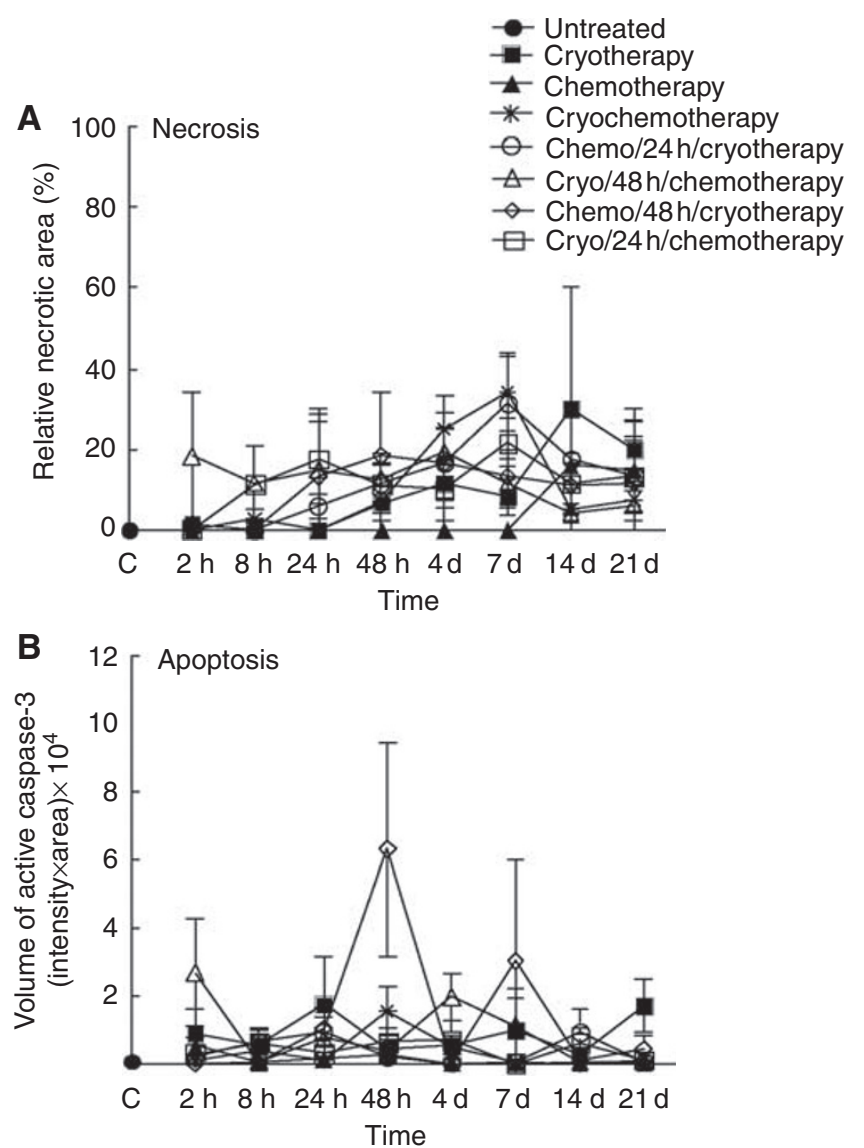

Figure 2 Kinetics of cell death after individual, simultaneous and sequential combination of cryotherapy and VNB treatment. (A) The relative percentage of necrotic area (y axis) was determined in tumour samples collected at various times ( $x$ axis) after the end of treatments by Haematoxylin-Eosin-Saffron staining. Points and vertical bars represent the means \pm s.e.m. of three independent samples (three mice). $C$ means untreated control tumours at time 0 . (B) The amount of apoptotic cells is reported as volume values (intensity $(\%) \times$ area $\left(\mu \mathrm{m}^{2}\right)$ ) determined after immunohistochemical staining (as described in Materials and Methods) in tumour samples collected at various times ( $x$ axis) after the end of treatments. Points and vertical bars represent the means \pm s.e.m. of three independent samples (three mice). C means untreated control tumours at time 0. Statistical analyses are provided in Supplementary Data.

VNB, $48 \mathrm{~h}$ before cryotherapy, compared with untreated or single agent-treated mice (Figure 4 ).

\section{DISCUSSION}

The idea of combining cryosurgery and chemotherapy in cancer treatment is not novel, but their optimisation needs further evaluation. The first study was reported by Benson in 1975 when he showed, in patients with oral cancer, that 5-FU was more efficient when administered after cryotherapy, with its increased accumulation in tumours immediately after cryosurgery (Benson, 1975). Later, Ikekawa et al (1985) confirmed, in a model of melanoma cells grafted into mice, that some drugs are trapped rapidly in tumours immediately after cryosurgery. This drugtrapping effect was observed only when the drugs were injected after cryosurgery because of microcirculation disturbance and particularly local vascular stasis induced with freezing. Indeed, it is well documented that cryotherapy elicits two major effects, a physical effect, which is immediate and known as 'direct cell injury', corresponding to the formation of ice crystals and a vascular effect, which is mostly delayed (Gage and Baust, 1998). Similarly, Homasson et al (1992) observed increased bleomycin uptake (about 30\%) in tumours when it was injected $2-6 \mathrm{~h}$ after cryosurgery in patients with inoperable lung cancer. More recently, in vitro studies have reported such a benefit of combined treatments, but some discrepancies appeared with the sequence of treatment. For instance, Clarke et al $(1999,2004)$ found that chemotherapy was more efficient when followed by cryosurgery in a model of renal carcinoma cells. Indeed, they documented that simultaneous 5-FU addition or administration 2 days after cryotherapy resulted in synergistic lethality, although many cells survived these treatments. However, when the cells were treated with 5-FU 2 days before cryosurgery, there was an apparent complete loss of cell viability. We have previously shown increased cell death after cryosurgery followed by chemotherapy in a model of A549 lung adenocarcinoma cells in SCID mice (Forest et al, 2005a, b; Forest et al, 2006). To optimise the efficiency of these combination treatments, we now report the effects of different administration schedules on tumour growth, cell death induction and apoptosis signalling. Our data suggest that $48 \mathrm{~h}$ sequential rather than simultaneous cryotherapy with VNB enhances the tumour response, with no significant difference seen within the sequence of administration. Our data also show that a $24 \mathrm{~h}$ sequential cryotherapy with VNB does not statistically give more benefit than simultaneous therapies, although some individual mice did respond well. In addition, our data argue that VNB administration $48 \mathrm{~h}$ before cryotherapy will provoke apoptosis more efficiently in these tumours, an effect associated with strong activation of the BH3-only Puma, Noxa and Bim.

It is established that cryosurgery, in addition to necrosis, could trigger apoptosis, and a few studies have implicated the mitochondrial pathway of apoptosis (Hanai et al, 2001; Baust et al, 2004). Clarke et al (2004) showed that freezing and chemotherapy differentially activated Bcl-2 family members in a human prostate cancer cell line. Freezing resulted in Bcl-2 upregulation, whereas chemotherapy triggered increased Bax expression. Freezing-induced $\mathrm{Bcl}-2$ upregulation could be prevented by the addition of 5-FU or cisplatin. Yang et al (2003) further confirmed that cryo-ended apoptosis was associated with mitochondrial dysfunction in four human colorectal cancer cell lines with variations in the induction of the antiapoptotic Bcl-2 and Bcl-xL, and the proapoptotic Bax, Bcl-xS, Bad and Bak proteins. Nevertheless, the Bcl-2/Bax protein ratio was inversely correlated with the apoptotic rate, an effect independent of p53 status in these lines.

In our study, despite variations among mice, no apparent differences in the amount of necrosis or apoptosis were observed among most combination protocols tested, except in the sequential treatment schedule where VNB was administered $48 \mathrm{~h}$ before cryotherapy. In this sequential treatment schedule, the number of apoptotic cells was significantly augmented $48 \mathrm{~h}$-post combined treatment, and correlated with heightened expression of the $\mathrm{BH} 3-$ only Puma, Noxa and Bim, at both the mRNA and protein levels. No significant change in Bax, Bcl-xL and Bcl-2 mRNA expression was evident, whereas $\mathrm{Mcl}-1$ expression increased only slightly to a much lower level than BH3-only mRNAs. Strikingly, no significant change in gene expression was seen in tumour samples from mice subjected to other protocols, although some benefit was apparent on tumour growth. Different hypotheses will be tested in future studies. First, we focused our expression study on a few Bcl-2 members, and investigation into other family members should be conducted. Second, other signalling pathway of apoptosis should be explored, including cell death receptor pathways. Finally, the importance of cytostatic effects, including growth arrest and premature senescence (Schmitt, 2007) where tumour cells are not 

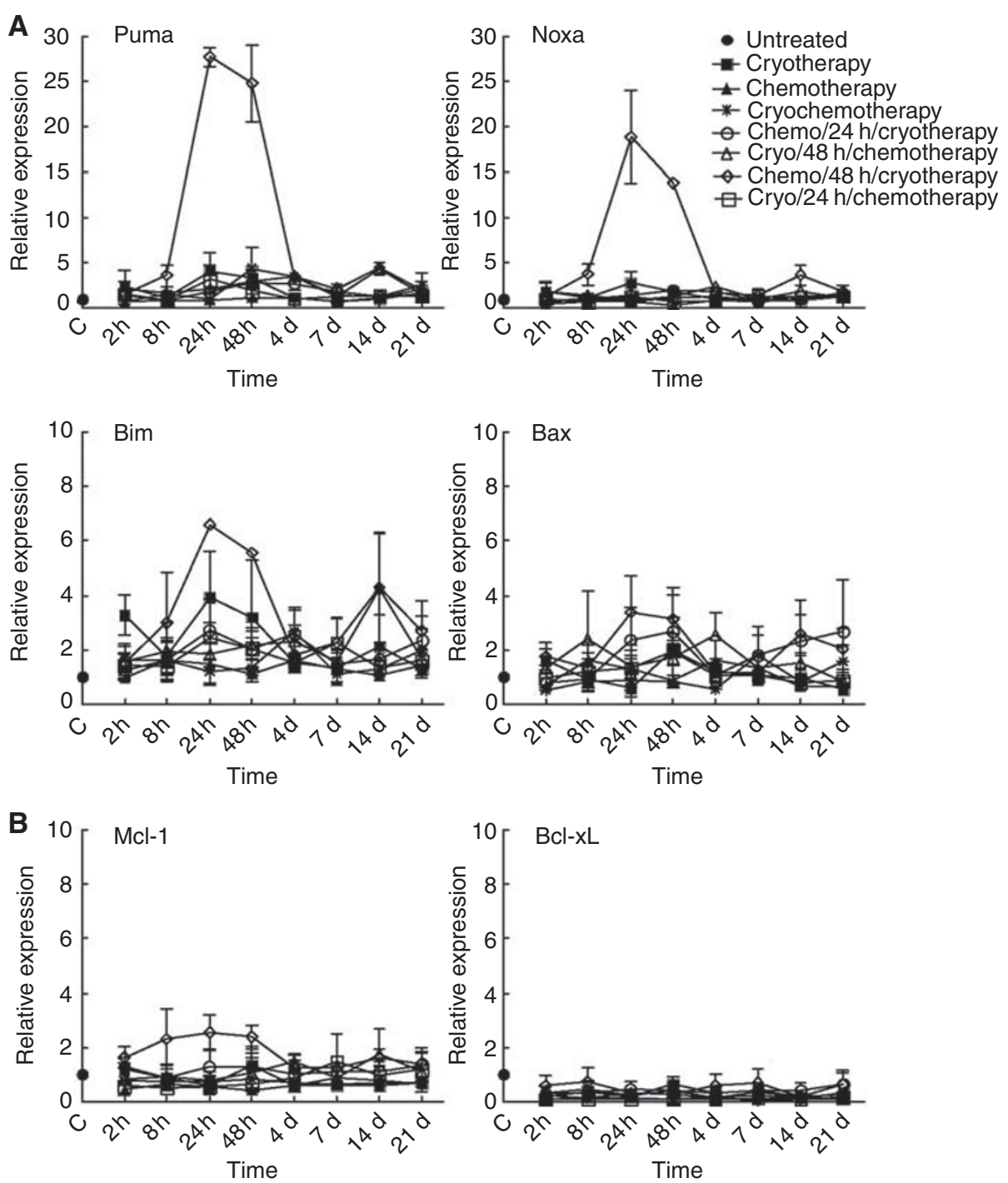

Figure 3 Relative quantitation of mRNA levels by QRT-PCR. (A) Relative expression of proapoptotic Puma, Noxa, Bim-EL and Bax mRNAs, and (B) antiapoptotic $\mathrm{Mcl}-\mathrm{I}$ and $\mathrm{Bcl}-\mathrm{xL}$ mRNAs, in tumour samples collected at various times ( $x$ axis) after the end of individual, simultaneous and sequentia combination treatments. The results are expressed as expression relative to untreated tumour samples. Points and vertical bars represent the means \pm s.e.m. of three independent samples (three mice), each analysed in duplicate. $\mathrm{C}$ means untreated control tumours at time 0 . Statistical analyses are provided in Supplementary Data.

dying, but stop proliferating, needs further attention. Our data revealed that TVs after the $48 \mathrm{~h}$ sequential schedule are nearly similar to TVs at day 0 of untreated mice, suggesting that cytostatic effects are important in this model.

Variations among individual mice were also observed in this study. Indeed, individual mice will have their own intrinsic variations and responses to treatment, but in vivo tumour models also have their own experimental challenges. It has been well established that tissue response to freezing is inhomogeneous as the influence of cold is spherical and decreases as a function of cryoprobe impact site. While producing tumour sample sections for microscopy, although special care was taken to cut them along the axis of the cryoprobe impact site, sample preparations could vary. Quantitative real-time PCR was also carried on tissues extracted along the same axis. Thus, a global analysis of the tumours rather than different areas relative to the cryoprobe impact site was carried out. Finally, with an in vivo model, tissue samples are collected at specific, fixed time points. It is, therefore, hard to investigate in detail a dynamic event or process. Perhaps, apoptotic cell phagocytosis by neighbouring cells is very dynamic and rapid, erasing traces of apoptosis in some tumour samples collected at fixed time points.

In conclusion, our data indicate that $48 \mathrm{~h}$ sequential rather than simultaneous or individual administration of cryotherapy with VNB in future cancer cryochemotherapy schedules enhances the tumour response. Our results also argue that VNB administration $48 \mathrm{~h}$ before cryotherapy will provoke apoptosis more efficiently in these tumours, an effect associated with Puma, Noxa and Bim-EL upregulation. However, we cannot extent our findings to other lung cancer models (epidermoid carcinoma instead of adenocarcinoma) or predict whether the same signalling pathways would be triggered with other chemotherapeutic agents (for instance if we use cisplatin instead of VNB). Therefore, further investigations are required for a better understanding of cryotherapy mechanisms of action and its biological actions at the tissue, cellular and molecular levels; but this study can represent a helpful starting 


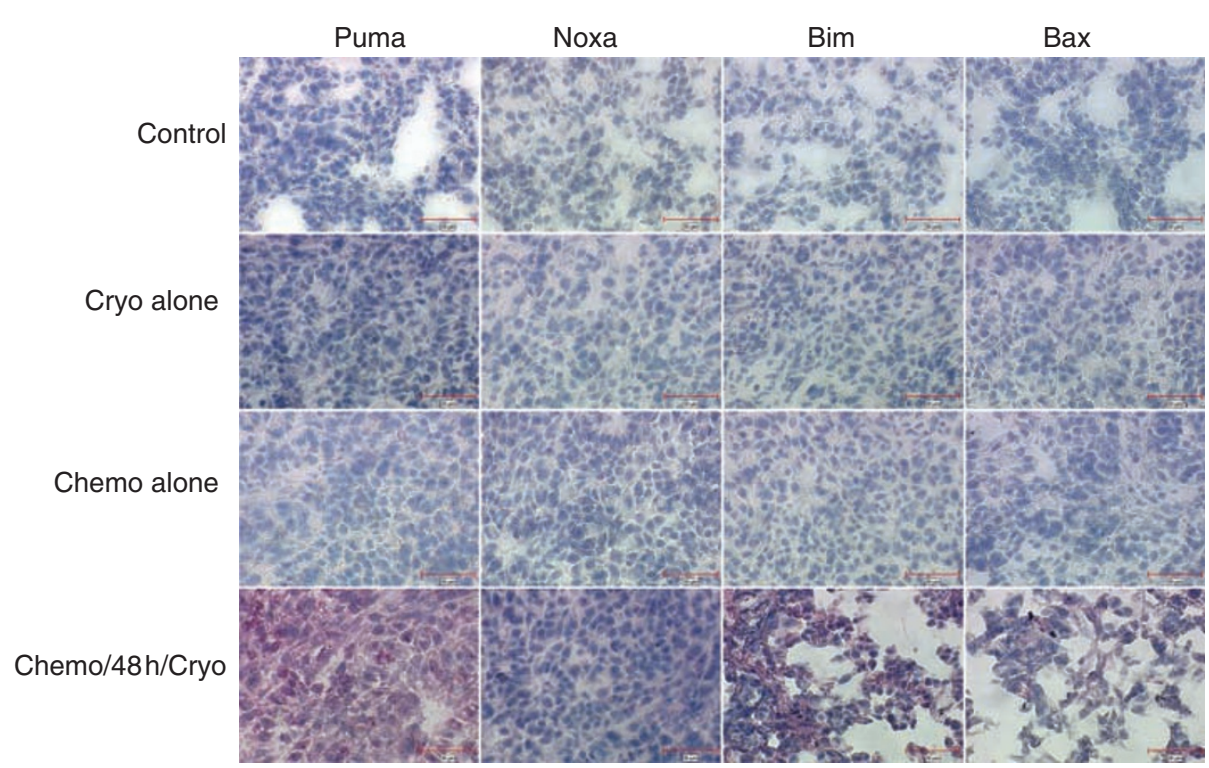

Figure 4 Protein expression in tumour samples after individual and sequential treatment schedules. Puma, Noxa, Bim and Bax protein expression levels were visualised by immunohistochemical staining, in tumour samples obtained $48 \mathrm{~h}$ post-treatment. The treatment protocols are indicated in the left margin. Micrographs are representative of three (Puma, Noxa) or two (Bim, Bax) independent tumour samples. Inset intervals: $30 \mu \mathrm{m}$ scale.

point. Better knowledge of the pathways of cell death, growth arrest and premature senescence induced by cryotherapy in combination with chemotherapy will lead to further optimisation of these combination schedules to achieve a better clinical response.

\section{ACKNOWLEDGEMENTS}

We thank Mr Ovid Da Silva (Research Support Office, Centre de recherche, CHUM) for his editorial work on this manuscript. This study was supported by Fonds de la recherche en bronchoscopie to RJF, CHUMHôpital Notre-Dame, Montréal (QC) H2 L 4M1, Canada.

Financial disclosures

No financial disclosures from any authors.

Supplementary Information accompanies the paper on British Journal of Cancer website (http://www.nature.com/bjc)

\section{REFERENCES}

Adams JM, Cory S (2007) The Bcl-2 apoptotic switch in cancer development and therapy. Oncogene 26: $1324-1337$

Baust JG, Gage AA, Clarke D, Baust JM, Van Buskirk R (2004) Cryosurgery a putative approach to molecular-based optimization. Cryobiology 48: $190-204$

Benson JW (1975) Combined chemotherapy and cryosurgery for oral cancer. Am J Surg 130: 596-600

Clarke DM, Hollister WR, Baust JG, Van Buskirk RG (1999) Cryosurgical modeling: sequence of freezing and cytotoxic agent application affects cell death. Mol Urol 3: 25-31

Clarke DM, Baust JM, Van Buskirk RG, Baust JG (2001) Chemo-cryo combination therapy: an adjunctive model for the treatment of prostate cancer. Cryobiology 42: 274-285

Clarke DM, Baust JM, Van Buskirk RG, Baust JG (2004) Addition of anticancer agents enhances freezing-induced prostate cancer cell death: implications of mitochondrial involvement. Cryobiology 19: 45-61

Forest V, Peoc'h M, Campos L, Guyotat D, Vergnon JM (2005a) Effects of cryotherapy or chemotherapy on apoptosis in a non-small-cell lung cancer xenografted into SCID mice. Cryobiology 50: 29-37

Forest V, Peoc'h M, Ardiet C, Campos L, Guyotat D, Vergnon JM (2005b) In vivo cryochemotherapy of a human lung cancer model. Cryobiology 51: $92-101$

Forest V, Peoc'h M, Campos L, Guyotat D, Vergnon JM (2006) Benefit of a combined treatment of cryotherapy and chemotherapy on tumour growth and late cryo-induced angiogenesis in a non-small-cell lung cancer model. Lung Cancer 54: 79-86

Gage AA, Baust J (1998) Mechanisms of tissue injury in cryosurgery. Cryobiology 37: $171-186$

Hanai A, Yang WL, Ravikumar TS (2001) Induction of apoptosis in human colon carcinoma cells HT29 by sublethal cryo-injury: mediation by cytochrome c release. Int J Cancer 93: 526-533

Homasson JP, Pecking A, Roden S, Angebault M, Bonniot JP (1992) Tumour fixation of bleomycin labeled with 57 cobalt before and after cryotherapy of bronchial carcinoma. Cryobiology 29: $543-548$

Ikekawa S, Ishihara K, Tanaka S, Ikeda S (1985) Basic studies of cryochemotherapy in a murine tumour system. Cryobiology 22: $477-483$

Korpan NN (2001) Indications of cryosurgery in pulmonology. In: Basics of cryosurgery. Springer-Verlag:Vienna, Austria, pp 181-187

Korsmeyer SJ, Shutter JR, Veis DJ, Merry DE, Oltvai ZN (1993) Bcl-2/Bax: a rheostat that regulates an anti-oxidant pathway and cell death. Semin Cancer Biol 4: $327-332$

Le Pivert P, Haddad RS, Aller A, Titus K, Doulat J, Renard M, Morrisson DR (2004) Ultrasound guided combined cryoablation and microencapsulated 5-fluorouracil inhibits growth of human prostate tumours in xenogenic mouse model assessed by luminescence imaging. Technol Cancer Res Treat 3: 135-142

Maiwand MO, Homasson JP (1995) Cryotherapy for tracheobronchial disorders. Clin Chest Med 16: 427-443

Mir LM, Rubinsky B (2002) Treatment of cancer with cryochemotherapy. Br J Cancer 86: $1658-1660$ 
Oda E, Ohki R, Murasawa H, Nemoto J, Shibue T, Yamashita T, Tokino T, Taniguchi T, Tanaka N (2000) Noxa, a BH3-only member of the bcl-2 family and candidate mediator of p53-induced apoptosis. Science 288: $1053-1058$

Pfaffl MW (2001) A new mathematical model for relative quantification in real-time RT-PCR. Nucleic Acids Res 29: 2002 - 2007

Schmitt CA (2007) Cellular senescence and cancer treatment. Biochim Biophys Acta 1775: 5-20,

Vergnon JM (1999) Cryothérapie endobronchique: techniques et indications. Rev Mal Respir 16: 619-623
Willis SN, Fletcher JI, Kaufmann T, van Delft MF, Chen L, Czabotar PE, Ierino H, Lee EF, Fairlie WD, Bouillet P, Strasser A, Kluck RM, Adams JM, Huang DC (2007) Apoptosis initiated when BH3 ligands engage multiple Bcl-2 homologs, not Bax or Bak. Science 315: $856-859$

Yang WL, Addona T, Nair DG, Qi L, Ravikumar TS (2003) Apoptosis induced by cryo-injury in human colorectal cancer cells is associated with mitochondrial dysfunction. Int J Cancer 103: 360 - 369

Yu J, Zhang L, Hwang PM, Kinzler KW, Vogelstein B (2001) Puma induces the rapid apoptosis of colorectal cancer cells. Mol Cell 7: 673-682 